\title{
Progesterone enhances cytokine-stimulated nitric oxide synthase II expression and cell death in human breast cancer cells
}

\author{
Fatima Bentrari $^{1}$, Laurent Arnould ${ }^{1,2}$, Antony P Jackson ${ }^{3}$, Jean-Francois Jeannin ${ }^{1}$ and \\ Alena Pance ${ }^{1,3}$ \\ ${ }^{1}$ EPHE INSERM 517, Faculty of Medicine, University of Bourgogne, Dijon, France; ${ }^{2}$ Department of \\ Pathology, Centre G-F Leclerc, Dijon, France and ${ }^{3}$ Department of Biochemistry, University of Cambridge, \\ Cambridge, UK
}

\begin{abstract}
The presence of hormone receptors is related to survival outcome in breast cancer. Previous results from our laboratory established a correlation between the presence of nitric oxide synthase II (NOSII) and nitric oxide (NO) production with progesterone receptors in a series of human breast tumours. Furthermore, this was directly related to a lower tumour grade and a lower proliferation rate of the tumour cells. To examine these results in further detail, the effect of progesterone $(\mathrm{Pg})$ and $17 \beta$-oestradiol $(\mathrm{E2})$ on NOSII expression was analysed in the human breast cancer cell line MCF-7. By Northern blot and promoter activity, we show that a cytokine mix (TNF- $\alpha$, IL- $\beta$, and IFN- $\gamma$ ) induces NOSII transcription after $6 \mathrm{~h}$ stimulation. In the absence of cytokines, neither hormone affects NOSII expression. However, Pg but not E2, enhances cytokine-induced NOSII transcription as well as NO synthesis, mainly by cooperation with gamma-interferon. The increase in NO accumulation in the media induced by addition of $\mathrm{Pg}$ to the cytokine treatment significantly increases cell death, mainly accounted for by apoptosis, as compared to the effect of cytokines alone. Our findings help clarify the role of steroid hormones in NOSII expression as well as the effect on cell viability and may suggest novel approaches towards hormonotherapy and the treatment of cancer.
\end{abstract}

Laboratory Investigation (2005) 85, 624-632, advance online publication, 21 March 2005; doi:10.1038/labinvest.3700267

Keywords: NOSII; progesterone; transcription; breast cancer; apoptosis; nitric oxide

Endogenous nitric oxide (NO) is produced in cells by the nitric oxide synthases (NOS), which exist as three isoforms. The calcium-dependent endothelial (eNOS or NOSIII) and neuronal ( $n N O S$ or NOSI) enzymes are constitutively present in various cell types and produce low levels of short acting NO. ${ }^{1}$ The third isoform (iNOS or NOSII) is induced in most cell types only in response to external stimuli such as bacterial lipopolysaccharides (LPS) or diverse cytokines, and generates high sustained levels of $\mathrm{NO}^{2} \mathrm{NO}$ is a transcellular messenger that plays an important role in diverse physiological processes such as vascular homeostasis, immunity and neurotransmission. The effect of endogenous NO on cellular homeostasis is highly dependent on

Correspondence: Dr A Pance, MSc, PhD, Department of Biochemistry, Hopkins Building, University of Cambridge, Tennis Court Road, Cambridge CB2 1QW, UK.

E-mail: a.pance@bioc.cam.ac.uk

This work is dedicated to Jeannick Lohr.

Received 29 November 2004; revised 24 January 2005; accepted 25 January 2005; published online 21 March 2005 the level of its synthesis. Thus, at low levels it can promote cell proliferation and angiogenesis whereas at high concentrations, such as those produced by NOSII, it can lead to cell damage and death. ${ }^{3}$ Therefore, it is important to understand the regulation of the NOS enzymes in order to unravel the role of NO in physiological and pathological conditions. So far it is known that expression of NOSII is regulated mainly at the transcriptional level, although additional post-transcriptional and posttranslational controls have been documented. ${ }^{4}$ The most important transcription factors required for NOSII expression are nuclear factor kappa B $(N F \kappa B)^{5}$ and activator protein $1(A P-1){ }^{6}$ These transcription factors are induced or activated by cytokines such as tumour necrosis alpha $(T N F-\alpha)$, interleukin-1beta $(I L-1 \beta)$, gamma interferon $(I F N-\gamma)$ or LPS. ${ }^{7}$ However, differences in the extent of cytokine-stimulated NOSII expression have been observed between diverse cell types. ${ }^{8}$ This raises the question of how NOSII expression is regulated in different cells and what additional factors contribute to its transcriptional regulation. 
Various studies have reported the presence of all three isoforms of NOS in a variety of tumours of diverse origin. However, the precise effects of NO on tumour growth are complex and remain largely unclear. Several lines of research have indicated that NO may promote tumour growth at low levels, ${ }^{9}$ while having a cytotoxic effect at high concentrations that abrogates both tumorigenicity and the formation of metastasis. ${ }^{10}$ Thus in human breast cancer, initial studies suggested a higher NOSII activity in less differentiated carcinomas, detecting expression of the enzyme predominantly in macrophages. More recently, the level of NOSII expression in the cancer cells has been inversely correlated with tumour grade in breast and colon carcinomas, ${ }^{11-13}$ and correlated with a higher apoptotic index. ${ }^{14}$ This suggests that NO could play a dual role in cancer, depending on the cell type and the concentration of NO produced, a conclusion strengthened by the finding that NOSII activity in cells that show increased tumorigenicity is at least an order of magnitude lower than the activity associated with cytotoxicity and apoptosis. ${ }^{9}$

It is well known that the status of steroid hormone receptors is an important prognosis factor in breast cancer. ${ }^{15,16}$ Thus oestrogen receptor $(E R)$ negative tumours generally show poorer prognosis than ER-positive tumours and the worst scenario arises when both oestrogen and progesterone receptors are absent. ${ }^{17}$ These findings led to the establishment of hormonotherapy as an important strategy for the treatment of diverse types of cancer, as well as an indicator of the response in breast cancer patients. ${ }^{18,19}$ Steroid hormones and their receptors have been correlated with the NO pathway in many systems. Thus, modulation of the constitutive NOSIII by hormonal status was suggested in invasive breast carcinomas. ${ }^{20}$ Oestradiol has been demonstrated to induce $N^{2} I^{21}{ }^{21}$ while inhibiting NOSII in different systems. ${ }^{22}$ But little is known about the effect of these hormones and their receptors on NOSII expression and activity.

We have previously shown that high levels of NOSII activity are correlated with lower in situ cell proliferation and lower tumour grade in breast carcinoma. We also found a correlation between NOSII expression and the presence of progesterone $(\mathrm{Pg})$ receptors. ${ }^{11}$ Here we show that Pg potentiates the transcriptional activation of NOSII by cytokines, mainly by cooperation with IFN- $\gamma$. Furthermore, we demonstrate that the expression of NOSII and the associated high production of NO induce cell death and that this effect is significantly increased by the presence of $\mathrm{Pg}$, mainly through an increase of apoptosis. Our data help build a molecular explanation for the observed relationship between hormone responsiveness and tumour phenotype in breast cancer.

\section{Materials and methods}

\section{Cell Culture}

Human breast cancer MCF-7 cells were maintained in D-MEM supplemented with $10 \%$ foetal calf serum and $2 \mathrm{mM}$ glutamine. The absence of mycoplasma contamination was verified regularly using the Mycotect kit (GIBCO BRL, Cergy Pontoise, France). Before stimulation, cells were depleted of hormones and growth factors in D-MEM without phenol red supplemented with $1 \%$ charcoal stripped foetal calf serum (Sigma-Aldrich, France) and glutamine for $24 \mathrm{~h}$ (stripped medium). Cells were then stimulated in the same medium.

\section{Cell Transfection and Reporter Gene Assay}

Cells were plated on six-well plates at such a density as to obtain a confluent monolayer $\left(1 \times 10^{6}\right.$ cells per $\mathrm{cm}^{2}$ ), and transfected with FuGENE 6 as indicated by the manufacturer (Qiagen Courtaboeuf, France). A pXP2 plasmid containing $7 \mathrm{~kb}$ of the NOSII promoter, kindly provided by Michael de Vera et $a l,{ }^{23}$ was used to examine transcriptional activity. Cells were routinely cotransfected with a TK-renilla luciferase plasmid, in order to normalise for transfection efficiency. After an overnight incubation, the transfection reagent was removed and fresh controlled medium added. The following day, cells were stimulated with a cytokine mix (CM) of $I L-1 \beta(3 \mathrm{ng} / \mathrm{ml}), T N F-\alpha(100 \mathrm{ng} / \mathrm{ml})$ and $I F N-\gamma(300 \mathrm{U} /$ $\mathrm{ml}$ ) for the appropriate length of time, usually $6 \mathrm{~h}$ and/or $17 \beta$-oestradiol (E2) (1 nM; Sigma-Aldrich, France) or progesterone (1 nM; Sigma-Aldrich, France). The dual luciferase reporter assay kit from Promega (Madison, WI, USA) was used to determine transcriptional activation following the users manual. In all, $10 \mu \mathrm{g}$ of total protein were used to perform the dual assays, measuring luciferase and renilla activity in a luminometer Lumat LB9507 (EG\&G Berthold, Bad Wilbad, Germany).

\section{RNA Extraction and Northern Blot}

RNA isolation and Northern Blotting was performed as described previously. ${ }^{24} \mathrm{~A} 600 \mathrm{bp}$ fragment of the human NOSII cDNA (46-644) was used as a probe for NOSII detection. This fragment was obtained by enzymatic digestion of a pbluescript plasmid containing human hepatocyte NOSII cDNA, kindly donated by Michael de Vera. The sequence was compared with the two constitutive NOS isoforms to verify exclusive homology with NOSII. The fragment was purified and labelled using the Promega random primed DNA labelling kit following the corresponding protocol. The labelled probe was purified on a Sephadex 50 column (Boehringer, Mannheim, Germany). 


\section{Electrophoretic Mobility Shift Assay}

The assays were performed as described previously. ${ }^{25}$ The oligonucleotides were labelled with T4 polynucleotide kinase following the manufacturer's instructions and purified on sephadex 50 columns (Boehringer, Mannheim, Germany) to eliminate unicorporated radioactivity. The consensus $A P-1$ and $N F_{\kappa} B$ oligonucleotides were obtained from Promega (Madison, WI, USA). The specific antibodies to the $A P-1$ family and $N F \kappa B$ were purchased from Santa Cruz Biotechnology Inc. (CA, USA).

\section{Proliferation}

Cells were plated in 96-well plates at 10000 cells/ well in complete medium. After a 24-h incubation in depleted medium, they were stimulated as indicated for 24 and $48 \mathrm{~h}$. Following stimulation, the medium was changed adding ${ }^{3} \mathrm{H}$-thymidine $(1 \mu \mathrm{Ci} / \mathrm{ml})$ and incubating $18 \mathrm{~h}$. After two washes with phosphatebuffered saline (PBS), cells were lysed with $1 \mathrm{M}$ $\mathrm{NaOH}$ for $1 \mathrm{~h}$ at $50^{\circ} \mathrm{C}$ and the incorporated radioactivity counted in a liquid scintillation counter (Wallac 1409, EG\&G Instruments).

\section{Cell Mortality and Apoptosis}

Cells were treated for the indicated periods of time with either hormone alone or together with cytokines. After stimulation, cells were recovered by trypsinisation and centrifugation, resuspended in phosphate saline buffer. An aliquot was then stained with trypan blue counting the viable (clear) and dead (blue) cells on the light microscope using a cytometer, obtaining the percentages of live and dead cells for each treatment. A second aliquot was used to assess characteristic apoptotic morphological changes. For this purpose, each sample was spread on a glass plate, washed once with phosphate saline buffer and fixed with $3 \%$ paraformaldehyde. The nuclei were stained with the Hoechst 33342 reagent $(1 \mathrm{mg} / \mathrm{ml})$ for $30 \mathrm{~min}$ at room temperature and chromatin condensation was examined using a fluorescent microscope. A total of at least 200 cells in each sample were counted to determine frequency of apoptosis.

\section{NO Biosynthesis Determination}

Cells were plated in six-well plates at $5 \times 10^{5} /$ well in complete medium. They were depleted of hormones for $24 \mathrm{~h}$ and stimulated for $24 \mathrm{~h}$. The supernatants were recovered and the concentration of $\mathrm{NO}_{2}$ was determined by the Griess reaction. ${ }^{26}$ The $\mathrm{NO}_{3}$ were reduced to $\mathrm{NO}_{2}$ by the nitrate reductase and these were measured using the same system.

\section{Statistical Analysis}

The experimental values are expressed as the mean \pm standard error of the mean for a number of separate experiments, which is indicated in each case but is in all cases at least three. The Student's $t$-test was used for comparison between two groups or experimental conditions. Significant differences were considered for those probabilities $<5 \%$ $(P<0.05)$.

\section{Results}

\section{NOSII is Induced by Cytokines in MCF-7 Cells}

The human breast cancer cell line MCF-7 is a widely used model for the study of human breast cancer. Here, we use this cell line to examine the role of steroid hormones in the expression of NOSII. Under control conditions, MCF-7 cells do not express NOSII. However, stimulation with a CM containing $T N F-\alpha(100 \mathrm{ng} / \mathrm{ml}), I L-1 \beta(3 \mathrm{ng} / \mathrm{ml})$ and $I F N-\gamma(300 \mathrm{U} / \mathrm{ml})$, induces NOSII mRNA accumulation as detected by Northern blotting, which is maximal after $6 \mathrm{~h}$, decreasing thereafter (Figure 1a). These results were confirmed using a luciferase reporter gene containing the proximal $7 \mathrm{~kb}$ of the human NOSII promoter. The transcriptional activation of the NOSII promoter reflects the mRNA accumulation detected by Northern blot being maximal $6 \mathrm{~h}$ after cytokine stimulation (Figure 1b). Thus, this is the stimulation time used for the following transcription experiments.

\section{Progesterone Increases Cytokine-Induced Transcription of NOSII}

To study the effect of steroid hormones, it was first necessary to deplete the culture medium of steroids and other compounds such as phenol red that could mimic their activity. Thus, $24 \mathrm{~h}$ prior to the experiments, cells were changed into a phenol red-free medium supplemented with $1 \%$ charcoal-stripped foetal calf serum. Since culturing breast cells in charcoal-stripped serum has been reported to repress progesterone receptor $(P g R)$ expression after 5 days, ${ }^{27}$ we performed our experiments within this period and verified by enzyme-linked immunosorbent assay (ELISA) the presence of both steroid receptors in our culture conditions (Table 1). We then examined the effect of steroid hormones on NOSII transcription using the reporter gene system described above to measure promoter activity. For this purpose, cells were stimulated with physiological concentrations of Pg $(1 \mathrm{nM})$ and E2 (1 nM) with or without cytokines for $6 \mathrm{~h}$. There is no transcriptional activation in response to either hormone alone, but when added together with CM, Pg increases cytokine-stimulated promoter activity almost two-fold (Figure 2a). This effect is highly significant $(P<0.001)$. Consistent with these results, 

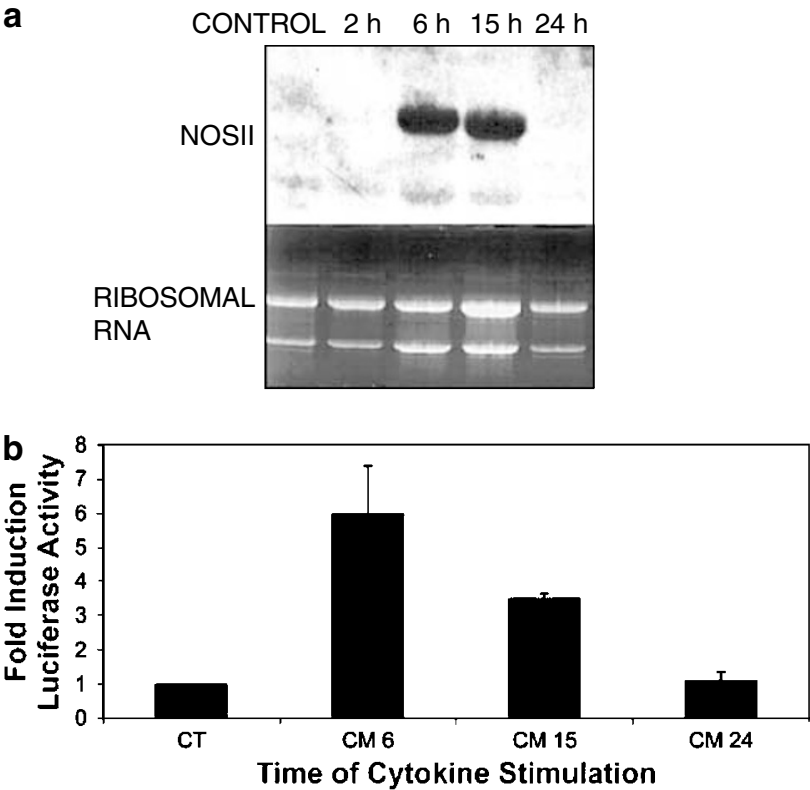

Figure 1 Transcription of NOSII in response to cytokine stimulation (CM) containing $I L-1 \beta, I F N-\gamma, T N F-\alpha$. (a) Northern blot for NOSII: total RNA was extracted from MCF-7 cells control (CT) or stimulated with CM for the indicated times. The blot was probed for NOSII as described in Materials and methods and is a representative result from three independent experiments. (b) Time course of transcriptional activity of NOSII promoter: MCF-7 cells were transiently transfected with the luciferase reporter gene containing the human NOSII promoter and transfection efficiency was normalised by cotransfection with TK-renilla as described in Materials and methods. Transfected cells were stimulated as indicated, and the results are expressed as fold induction of control nonstimulated cells (means \pm s.d. of six experiments).

Table 1 Hormone receptors in MCF-7 cells in the media used

\begin{tabular}{lcccc}
\hline MCF-7 & PgR (fM/mg) & s.d. & ER (fM/mg) & s.d. \\
\hline Standard medium & 167.5 & 9.19 & 221.5 & 12.02 \\
Stripped medium & 161 & 8.49 & 225.5 & 12.02
\end{tabular}

Standard medium: D-MEM with $10 \%$ foetal calf serum; Stripped medium: D-MEM without phenol red with $1 \%$ charcoal-tripped foetal calf serum.

cytokines stimulate NO production, which is further increased by the addition of Pg (Figure 2b). This additive effect of $\mathrm{Pg}$ on NO accumulation is significant $(P<0.005)$ and quantitatively consistent with an increase in protein expression. In contrast, E2 shows no effect on cytokine-stimulated NOSII promoter activity, on the contrary a decrease in NO accumulation is detected in the media of cells stimulated with CM and E2 (Figure 2a,b).

The effect of Pg on NOSII transcription induced by the $\mathrm{CM}$ is maximal at a concentration of $1 \mathrm{nM}$, as determined by a dose-response curve (not shown). Thus, all the following experiments were performed with Pg at this concentration, which is consistent with the physiological levels in breast tissue.
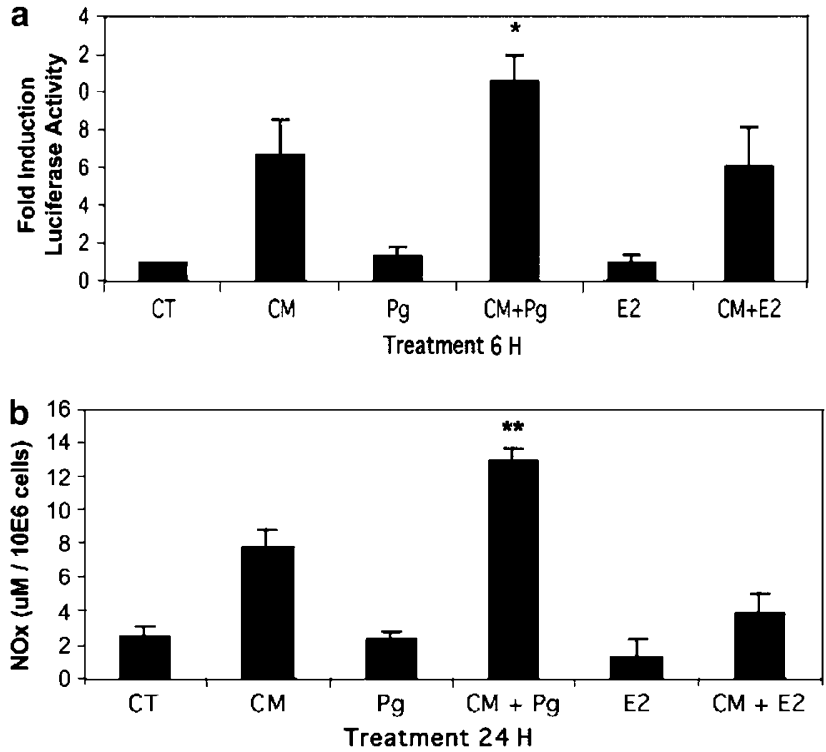

Figure 2 Effect of steroid hormones on NOSII transcriptional activation and NO production. MCF-7 cells were treated as indicated. (a) NOSII promoter activity upon treatment with hormones alone or together with cytokines for $6 \mathrm{~h}$ : cells were transiently transfected with the reporter gene as described before, and treated as indicated. Luciferase activity is reported as fold induction of nonstimulated cells (means \pm s.d. of four experiments) ${ }^{*} P<0.001$. (b) $\mathrm{NO}_{x}\left(\mathrm{NO}_{2}+\mathrm{NO}_{3}\right)$ accumulation after $24 \mathrm{~h}$ stimulation with either hormone with or without cytokines: $\mathrm{NO}_{x}$ in the media were determined by the Griess method as described. Values represent means \pm s.d. of four experiments. ${ }^{*} P<0.005$ vs CM.

We next examined the effect of Pg on each of the cytokines used to stimulate NOSII transcription to determine if $\mathrm{Pg}$ cooperates with a particular stimulus or adds to the overall stimulation by the mix of the three cytokines used. We and others have shown that the human NOSII promoter is poorly stimulated by each cytokine individually, needing the combination of all three for full transcriptional activation. Thus, as expected induction is very low with each cytokine alone, but addition of Pg slightly increases the effect in each case (Figure 3). Most noticeable is the effect on induction by $I F N-\gamma$ which is almost doubled by addition of Pg to a level close to that obtained with the combination of the three cytokines, that is, full transcriptional activation.

\section{Transcription Factors Involved in Hormone Stimulation}

In an attempt to unravel the mechanism of the effect of Pg, we studied the effect of the hormone on the transcription factors activated by cytokines that are implicated in the regulation of NOSII transcription. These are mainly $N F \kappa B^{5}$ and $A P-1,{ }^{6}$ so we examined the effect of both hormones on their DNA binding by electrophoretic mobility shift assay (EMSA). As 
expected, the CM induces $N F k B$ translocation to the nucleus and the DNA binding of the characteristic p50/p50 and p50/p65 dimers (Figure 4a), but there is no evidence for Pg enhancement of $N F \kappa B$ binding. $A P-1$ DNA binding is also induced by CM, while neither hormone has any additional effect (Figure 4b). The $A P-1$ family of transcription factors identified in MCF-7 cells correspond to junD, $c$-fos and a minor contribution of $f o s B$, as shown by a supershift with specific antibodies, while the other members of this family, such as Fra-2 are absent. These results strongly suggest that the Pg-enhancement of cytokine-stimulated NOSII expression cannot be caused by a direct interaction of the hormone with the cytokine-dependent transcription factors but imply an independent effect of Pg on promoter activity.

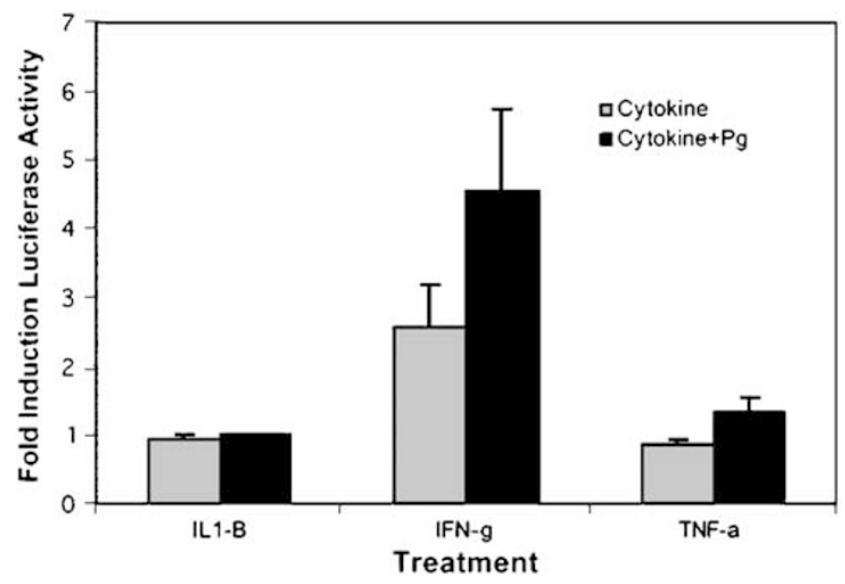

Figure 3 Effect of Pg on NOSII transcriptional activation by each cytokine individually. Cells were transiently transfected with the reporter gene of the NOSII promoter and stimulated as indicated for $6 \mathrm{~h}$. The resulting luciferase activity is expressed as fold induction relative to nonstimulated cells.

\section{Progesterone Increases Cytokine-Induced Cell Death in a NO-Dependent Manner}

To place our results into a broader physiological context, we studied both proliferative capacity and cell death in MCF-7 cells after treatment with CM with or without steroid hormones. Proliferation assays show a strong inhibition in the presence of the CM (Figure 5a) that is independent of the further addition of $\mathrm{Pg}$ (see Figure 5d). There is no significant effect of Pg alone on cell proliferation. By contrast, as previously reported, E2 stimulates proliferation (Figure 5a). This effect is mediated by the oestrogen receptor since it is inhibited by the known antagonist tamoxifen in a concentrationdependent manner (Figure 5b).

We examined the role of NO in cell proliferation using various NOS inhibitors to interfere with its synthesis. The general NOS inhibitor aminoguanidine (AG) greatly decreases proliferation in the absence of cytokines (Figure 5c), suggesting a role for basal NO production in driving the background rate of cell division. This is probably due to NOSIII (eNOS), since NOSII is not expressed under these circumstances. The role of NOSIII in this effect was examined with nitro-L-arginine methyl ester (L-NAME), which selectively inhibits this enzyme without affecting NOSII. The results shown in Figure 5c reveal an even stronger inhibitory effect of L-NAME than AG, confirming that basal proliferation as well as the proliferative effect of E2 are mediated by NO synthesised by NOSIII.

On the other hand, even though in the presence of $\mathrm{CM}$, proliferation is very low, a slight relief of this inhibition by AG can be seen in Figure 5d. However, these levels of proliferation do not reach the ones observed for untreated cells suggesting the participation of NO-independent mechanisms. Interestingly, AG has no effect on $\mathrm{CM}+\mathrm{E} 2$, which may be consistent with the results of Figure $2 \mathrm{~b}$ showing that

a CT $\quad \mathrm{CM} \quad \mathrm{Pg} \mathrm{CM}+\mathrm{Pg}$ E2 CM+E2 UP p50 p65

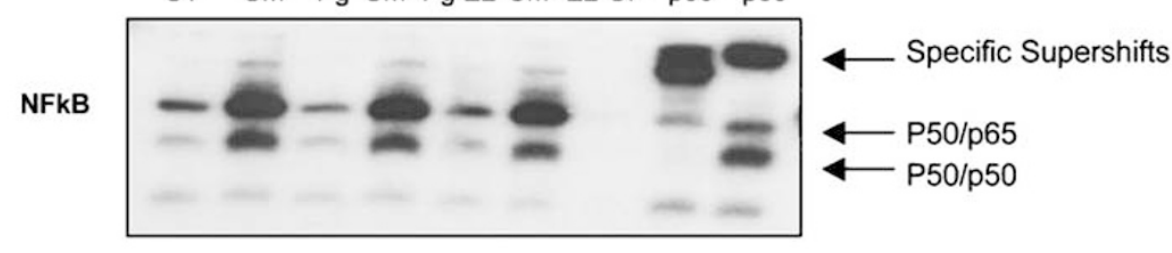

b

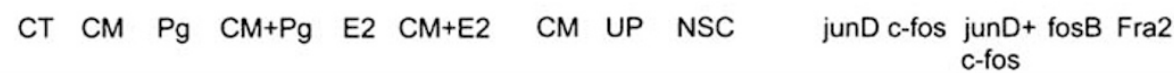

AP-1

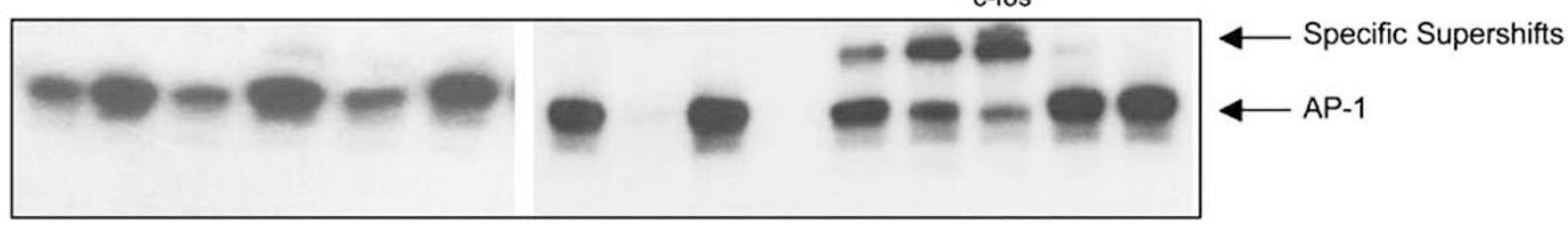

Figure 4 Effect of hormones on the main transcription factors implicated in NOSII transcriptional activation. Cells were treated as indicated for $2 \mathrm{~h}$ and EMSAs were performed as described in Materials and methods. (a) nuclear translocation and binding of $N F k B$. The p50 and p65 components of the complex were identified by supershift with specific antibodies. (b) AP-1 induction in MCF-7 cells and identification of the AP-1 members in the complexes with specific antibodies. UP: unlabelled probe; NSC: nonspecific competitor. 
E2 seems to inhibit NO synthesis induced by cytokines. This agrees with previous reports of NOSII inhibition by E2. ${ }^{22}$ L-NAME, on the contrary, does not relieve the cytokine inhibition but seems to add to the inhibitory effect, which could be the

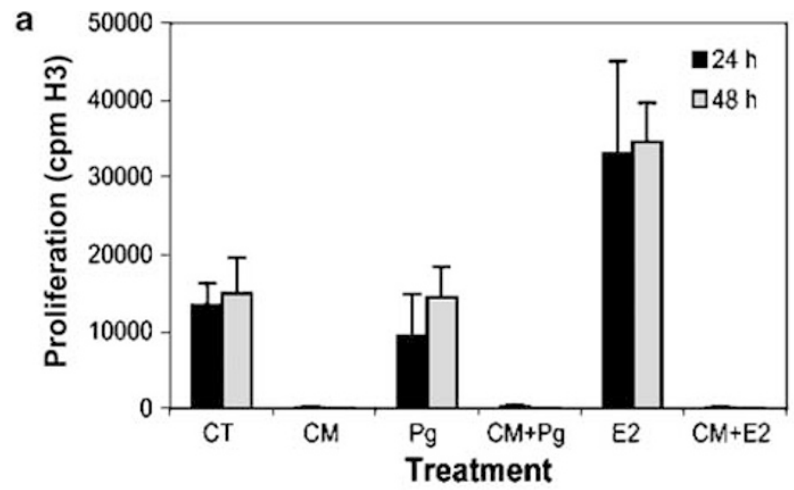

b
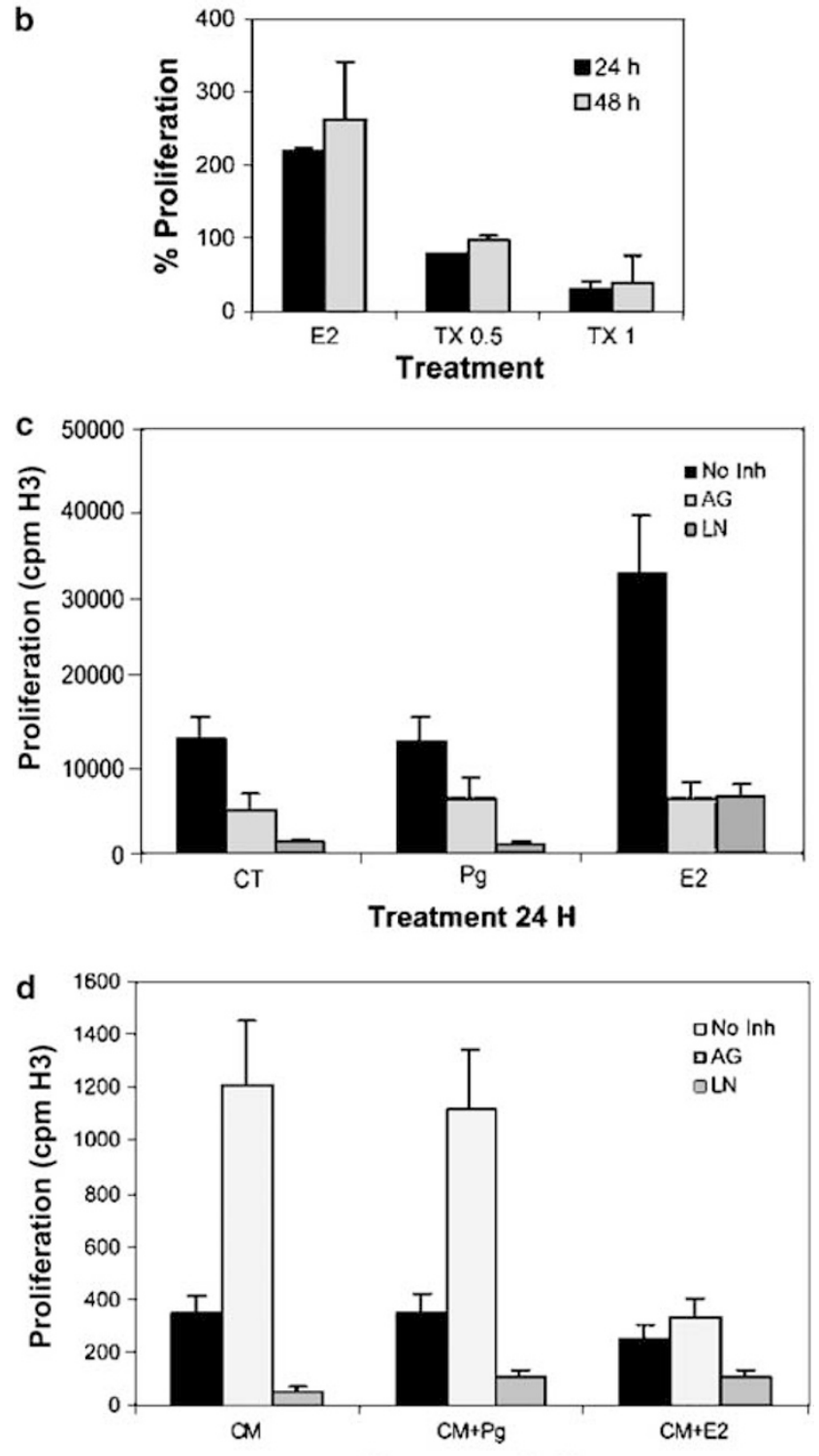

Treatment $24 \mathrm{H}$ result of inhibiting NOSIII and its proliferative effect. Taken together, these results indicate a minor role for NOSII in cytokine-induced cytostasis.

Cell death is not significantly increased by treatment with either hormone alone (Figure 6a) and the NOS inhibitor AG shows no effect in these conditions. The cytokine mix induces $30 \%$ cell death (adjusted to 100\% in Figure 6a) and this effect can be slightly inhibited by AG, suggesting a combination of NO-dependent as well as independent mechanisms. The specific NOSII inhibitor $1400 \mathrm{~W}$, decreases cell death to the same degree than AG (by $26 \%$ ), indicating that the high NO output by NOSII (Figure 6a) is involved but that other mechanisms play a major role in the general cytotoxic effect of cytokines. The addition of Pg to CM treatment greatly increases the CM-induced cell death (53\% increase relative to CM alone, $155 \%$ in Figure 6a). This effect is significant $(P<0.001)$ and consistent with the increase of NOSII transcription and NO production provoked by the addition of $\mathrm{Pg}$ to the CM (Figure 2). The inhibition by AG and $1400 \mathrm{~W}$ shows that the effect of $\mathrm{Pg}$ is mainly NOdependent and that this NO is produced by NOSII, since the level is similar to that obtained by inhibiting the CM treatment. E2 has no effect on cell death neither alone nor when added together with cytokines (Figure 6a).

The general cell death was compared with the frequency of apoptosis by Hoechst staining of treated cells and observation of chromatin condensation on the fluorescent microscope. This staining shows that while neither hormone alone has an effect (Figure 6b), the cytokines provoke apoptosis in a time-dependent manner, reaching $13 \%$ after $24 \mathrm{~h}$ of treatment. Consistent with the results of cell death, addition of Pg but not E2 causes a significant $(P<0.005)$ increase in the number of apoptotic cells compared to cytokine treatment alone. The effect of $\mathrm{Pg}$ is most noticeable after $24 \mathrm{~h}$, when the increase of apoptosis is approximately $35 \%$ compared to cytokines alone. The role of NOSII and its high NO output in the induction of apoptosis was confirmed by $1400 \mathrm{~W}$, which completely abrogates the effect of cytokines with and without either hormone (Figure 6c). These results indicate that $\mathrm{Pg}$ is capable of potentiating cytokine induction of NOSII expression, thus increasing NO-dependent apoptosis in breast cancer cells.

Figure 5 Effect of hormones and cytokines on proliferation of MCF-7 cells. Cells were processed and ${ }^{3} \mathrm{H}$-thymidine incorporation measured under the various conditions as described in Materials and methods. (a) Proliferation of control (CT) and treated cells measured after 24 and $48 \mathrm{~h}$. (b) Inhibition of the proliferative effect of E2 with tamoxifen (0.5 and $1 \mu \mathrm{M})$. (c) Inhibition of proliferation by the NOS inhibitor aminoguanidine (AG; $500 \mu \mathrm{M})$ and the NOSIII inhibitor L-NAME $(500 \mathrm{nM})$ in the absence of cytokines. (d) Effect of the two inhibitors AG and L-NAME on proliferation in the presence of cytokines with or without steroid hormones. All reported values represent means \pm s.d. of at least three experiments. 


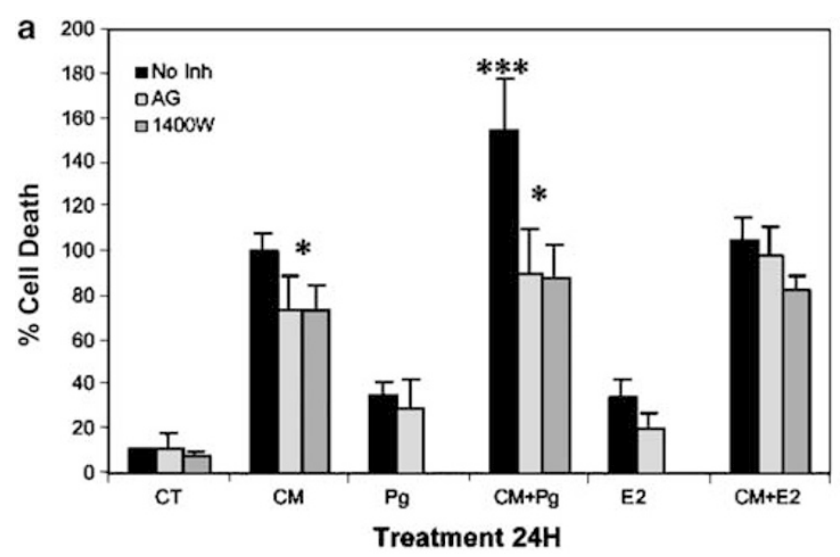

b
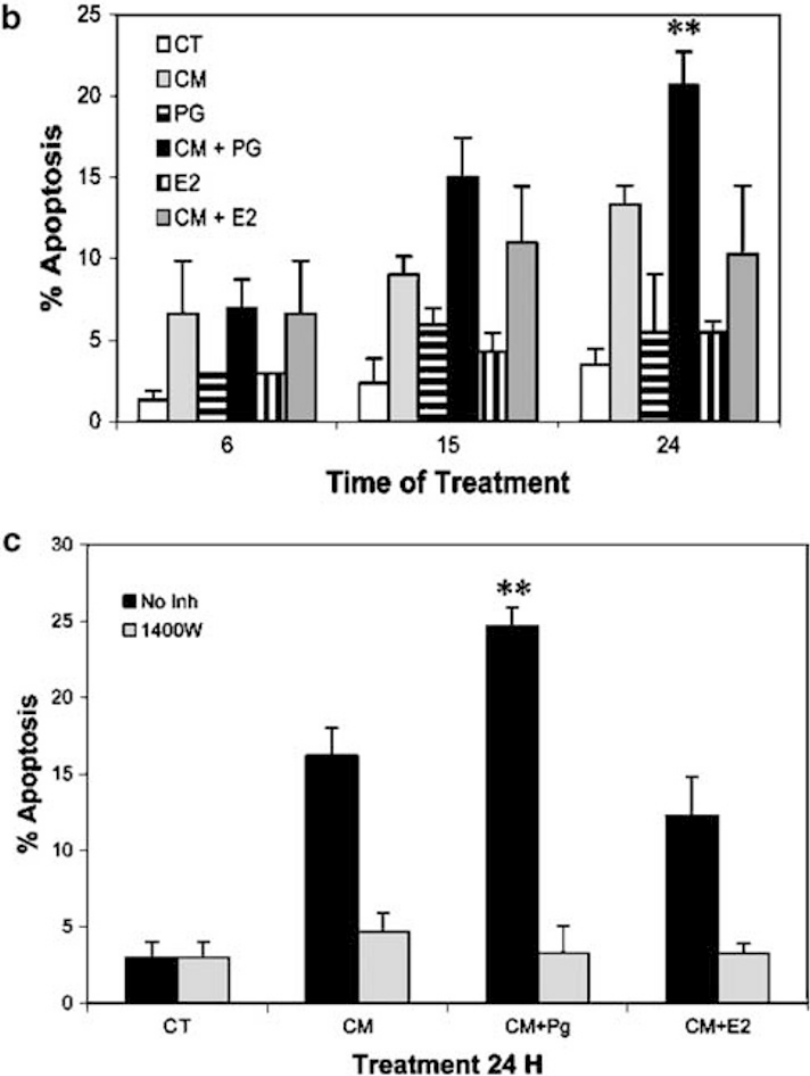

Figure 6 Cell death of MCF-7 cells upon treatment with hormones with or without cytokines. (a) Mortality revealed by trypan blue staining, after $24 \mathrm{~h}$ of the indicated treatment with the general NOS inhibitor aminoguanidine (AG; $500 \mu \mathrm{M}$ ) or the specific NOSII inhibitor $1400 \mathrm{~W}(25 \mu \mathrm{M})$ or without inhibitors (No inh). ${ }^{* *} P<0.001$ vs $\mathrm{CM} ;{ }^{*} P<0.01$ vs no inhibitor for both inhibitors used. (b) Time course of apoptosis induced by the different treatments. Apoptotic cells were stained with Hoechst 33342 and counted under the fluorescent microscope. ${ }^{* *} P<0.005$ vs CM. All reported values are means \pm s.d. of at least three experiments. (c) Effect of $1400 \mathrm{~W}$ on apoptosis induced by cytokines with or without hormones after $24 \mathrm{~h} .{ }^{* *} P<0.005$ vs CM.

\section{Discussion}

Our results show for the first time that Pg potentiates cytokine-induced transcription of NOSII in a human breast cancer cell line. It is well established that full activation of the human NOSII promoter requires a combination of cytokines, while response to individual stimulation with each cytokine alone is very low. ${ }^{24,28}$ Consistent with this mechanism, stimulation with $\mathrm{Pg}$ alone might not in itself be strong enough to drive transcription. Moreover, the fact that Pg does not enhance binding of the main transcription factors induced by cytokines, suggests a direct role of the $\mathrm{Pg}-\mathrm{Pg}$ receptor complex in the activation of the human NOSII promoter. We show that the main cooperative effect of $\mathrm{Pg}$ to induce NOSII transcription occurs with IFN- $\gamma$. This combination achieves the same level of NOSII transcription as the use of the three cytokines together, which may have important implications for therapeutic applications. Previous studies have reported that both Pg and E2 alone induce NOSII expression in different cell types in the uterus ${ }^{29}$ and that $\mathrm{Pg}$ stimulates NOSII expression and NO production in ovarian cancer cells. ${ }^{30}$ In contrast, other studies showed an inhibitory effect of Pg. Thus, Pg inhibited NOSII expression in murine macrophages, ${ }^{31}$ as well as in human colon enterocytes DLD-1 and Caco-2. ${ }^{32}$ However, it is known that macrophages do not express the Pg receptor. ${ }^{31}$ Furthermore, the presence of PgR was not demonstrated in the human enterocytes used in the latter study, ${ }^{32}$ but the fact that Pg inhibits NOSII in these cells post-transcriptionally strongly suggests a receptor-independent mechanism since the complex $\mathrm{Pg}-\mathrm{PgR}$ acts as a transcription factor. It is possible that in the absence of its receptor, Pg acts post-transcriptionally inhibiting NOSII, while when the receptor is present, it quickly sequesters the hormone, translocating it to the nucleus where the complex cooperates with other stimuli to induce NOSII transcription. The conclusion highlights the complexity of the effect of steroid hormones, which varies according to the cell type and its ultimate effect on NOSII expression critically depends on the presence of the receptor.

Treatment of MCF-7 cells with cytokines inhibits proliferation and the fact that this cytostatic effect shows low sensitivity to a NOS inhibitor, implicates alternative pathways. For example, it is known that $T N F-\alpha$ and IFN- $\gamma$ can directly induce G1 arrest by modulating cyclin expression and activation. ${ }^{33}$ On the other hand, the inhibition of the receptormediated proliferative effect of E2 by AG indicates the participation of a NOS enzyme. Inhibition by LNAME, a specific inhibitor of NOSIII, and the fact that NOSII is not expressed under these circumstances, indicate that the most likely candidate for this effect is NOSIII. Indeed it has previously been shown that E2 induces NOSIII expression ${ }^{21}$ and activity. ${ }^{34}$ Several studies have shown a positive correlation of NOSIII and oestrogen receptors in breast tumours ${ }^{35,36}$ and that the low level of NO produced by NOSIII is associated with endothelial proliferation. ${ }^{37}$ Furthermore, NOSIII is present in human malignant breast tumours where its expression correlates with tumour grade and is associated 
with increased breast cancer risk. ${ }^{38}$ It is known that the oestrogen-ER complex binds to the p85 $\alpha$ regulatory subunit of PI-3 kinase leading to the activation of $A k t$, and that this pathway is responsible for the phosphorylation of a C-terminal serine of NOSIII increasing its catalytic activity. Hence, it is possible that the oestrogen-ER complex can activate the release of NO by NOSIII and that this NO may contribute to tumour proliferation and survival. ${ }^{3}$

Beside their cytostatic effect, cytokines are highly cytotoxic for MCF-7 cells. At least two different pathways seem to be involved since AG and 1400W only slightly inhibit cytotoxicity. These pathways may induce diverse types of cell death including apoptosis, necrosis, etc; that will contribute to different degrees to the total cell death. On the one hand, the NO-independent death could be triggered by the cytokines used, such as $T N F-\alpha$ via the stimulation of its death domain receptor, while the 1400W/AG-sensitive pathway is consistent with the cytotoxicity caused by high levels of NO produced by NOSII in these conditions. These include inhibition of DNA and ATP synthesis, inhibition of cyclindependent kinase 2 and accumulation of p53 (for a review see $\mathrm{Xu}$ et $\mathrm{al}^{3}$ ). Thus, stimulation of apoptosis due to high NO levels has been described in human ovarian tumour cells. ${ }^{39}$ We show here that Pg increases cytokine-induced apoptosis and that the mechanism of this effect is a cooperation with cytokines to increase NOSII transcription and NO production. This is confirmed by the observation that both AG and 1400W inhibit the increase in cell death caused by Pg to the same levels as they inhibit the effect of cytokines alone. The reports that Pg induces apoptosis in malignant mesothelioma cells ${ }^{40}$ as well as in normal breast cells and various cancer cell lines ${ }^{41}$ further support this view. Furthermore, in agreement with the results presented here, the role of $\mathrm{Pg}$ in the induction of apoptosis via induction of NOSII to increase NO levels was shown in ovarian cancer cells, ${ }^{30}$ indicating a general function of $\mathrm{Pg}$ in this mechanism. In fact, several randomised studies and clinical trials have shown that treatment with progestogens led to significant though modest improvement of the relapse-free survival, ${ }^{42,43}$ and that inclusion of progestogens in hormone replacement therapy reduces the risk of recurrence, which is associated with an increase in the apoptosis/proliferation ratio within the tumour. ${ }^{44}$

The balance between cell death and cell proliferation determines tumour growth rate and even a small alteration in these parameters can be important for the expansion or regression of malignant tumours. Our results show that Pg can increase the inflammatory response against breast cancer cells by augmenting NO levels in the cells, thus leading to apoptosis. We suggest that pharmacological interventions to maximise the Pg-enhanced NOSII expression, perhaps in combination with IFN- $\gamma$ may be a plausible option for some forms of breast cancer.

\section{Acknowledgements}

AP was funded by the Conseil Regional de Bourgogne and the Fondation pour la Recherche Medicale. We are grateful to Dr Guy Brown for useful discussions and for providing the $1400 \mathrm{~W}$ NOSII inhibitor.

\section{References}

1 Forstermann U, Closs EI, Pollock JS, et al. Nitric oxide synthase isozymes. Characterization, purification, molecular cloning, and functions. Hypertension 1994; 23:1121-1131.

2 Forstermann U, Boissel JP, Kleinert H. Expressional control of the 'constitutive' isoforms of nitric oxide synthase (NOS I and NOS III). FASEB J 1998;12: 773-790.

$3 \mathrm{Xu}$ W, Liu LZ, Loizidou M, et al. The role of nitric oxide in cancer. Cell Res 2002;12:311-320.

4 Rao KM. Molecular mechanisms regulating iNOS expression in various cell types. J Toxicol Environ Health B Crit Rev 2000;3:27-58.

5 Taylor BS, de Vera ME, Ganster RW, et al. Multiple NFkappaB enhancer elements regulate cytokine induction of the human inducible nitric oxide synthase gene. J Biol Chem 1998;273:15148-15156.

6 Kristof AS, Marks-Konczalik J, Moss J. Mitogenactivated protein kinases mediate activator protein-1dependent human inducible nitric-oxide synthase promoter activation. J Biol Chem 2001;276:8445-8452.

7 Kleinert H, Wallerath T, Fritz G, et al. Cytokine induction of NO synthase II in human DLD-1 cells: roles of the JAK-STAT, AP-1 and NF-kappaB-signaling pathways. Br J Pharmacol 1998;125:193-201.

8 Jenkins NA, Rothe H, Gilbert DJ, et al. Mapping of the gene for inducible nitric oxide (NO) synthase of mouse macrophages to chromosome 11, close to Evi-2, nu, and Idd-4. Genomics 1994;19:402-404.

9 Jenkins DC, Charles IG, Thomsen LL, et al. Roles of nitric oxide in tumor growth. Proc Natl Acad Sci USA 1995;92:4392-4396.

10 Xie K, Huang S, Dong Z, et al. Transfection with the inducible nitric oxide synthase gene suppresses tumorigenicity and abrogates metastasis by K-1735 murine melanoma cells. J Exp Med 1995;181: 1333-1343.

11 Reveneau S, Arnould L, Jolimoy G, et al. Nitric oxide synthase in human breast cancer is associated with tumor grade, proliferation rate, and expression of progesterone receptors. Lab Invest 1999;79:1215-1225.

12 Ambs S, Merriam WG, Bennett WP, et al. Frequent nitric oxide synthase-2 expression in human colon adenomas: implication for tumor angiogenesis and colon cancer progression. Cancer Res 1998;58: 334-341.

13 Tschugguel W, Schneeberger C, Unfried G, et al. Expression of inducible nitric oxide synthase in human breast cancer depends on tumor grade. Breast Cancer Res Treat 1999;56:145-151.

14 Vakkala M, Kahlos K, Lakari E, et al. Inducible nitric oxide synthase expression, apoptosis, and angiogenesis in in situ and invasive breast carcinomas. Clin Cancer Res 2000;6:2408-2416. 
15 Knight WA, Livingston RB, Gregory EJ, et al. Estrogen receptor as an independent prognostic factor for early recurrence in breast cancer. Cancer Res 1977;37: 4669-4671.

16 Mason BH, Holdaway IM, Mullins PR, et al. Progesterone and estrogen receptors as prognostic variables in breast cancer. Cancer Res 1983;43:2985-2990.

17 McGuire WL, Osborne CK, Clark GM, et al. Steroid hormone receptors and carcinoma of the breast. Am J Physiol 1982;243:E99-E102.

18 Howell A, Barnes DM, Harland RN, et al. Steroidhormone receptors and survival after first relapse in breast cancer. Lancet 1984;1:588-591.

19 Alanko A, Heinonen E, Scheinin T, et al. Significance of estrogen and progesterone receptors, disease-free interval, and site of first metastasis on survival of breast cancer patients. Cancer 1985;56:1696-1700.

20 Vakkala M, Paakko P, Soini Y. eNOS expression is associated with the estrogen and progesterone receptor status in invasive breast carcinoma. Int J Oncol 2000;17:667-671.

21 Kleinert H, Wallerath T, Euchenhofer C, et al. Estrogens increase transcription of the human endothelial NO synthase gene: analysis of the transcription factors involved. Hypertension 1998;31:582-588.

22 Yallampalli C, Dong YL. Estradiol-17beta inhibits nitric oxide synthase (NOS)-II and stimulates NOS-III gene expression in the rat uterus. Biol Reprod 2000;63:34-41.

23 de Vera ME, Shapiro RA, Nussler AK, et al. Transcriptional regulation of human inducible nitric oxide synthase (NOS2) gene by cytokines: initial analysis of the human NOS2 promoter. Proc Natl Acad Sci USA 1996;93:1054-1059.

24 Pance A, Chantome A, Reveneau S, et al. A repressor in the proximal human inducible nitric oxide synthase promoter modulates transcriptional activation. FASEB J 2002;16:631-633.

25 Goldring CE, Reveneau S, Chantome A, et al. Heat shock enhances transcriptional activation of the murine-inducible nitric oxide synthase gene. FASEB J 2000;14:2393-2395.

26 Green LC, Wagner DA, Glogowski J, et al. Analysis of nitrate, nitrite, and [15N]nitrate in biological fluids. Anal Biochem 1982;126:131-138.

27 Moudgil VK, Dinda S, Khattree N, et al. Hormonal regulation of tumor suppressor proteins in breast cancer cells. J Steroid Biochem Mol Biol 2001;76: 105-117.

28 Kleinert H, Euchenhofer C, Fritz G, et al. Involvement of protein kinases in the induction of NO synthase II in human DLD-1 cells. Br J Pharmacol 1998;123: 1716-1722.

29 Huang J, Roby KF, Pace JL, et al. Cellular localization and hormonal regulation of inducible nitric oxide synthase in cycling mouse uterus. J Leukoc Biol 1995; 57:27-35.

30 Keith Bechtel M, Bonavida B. Inhibitory effects of 17beta-estradiol and progesterone on ovarian carcinoma cell proliferation: a potential role for inducible nitric oxide synthase. Gynecol Oncol 2001;82: 127-138.

31 Kohmura Y, Kirikae T, Kirikae F, et al. Onapristone (ZK299) blocks the suppressive effect of progesterone, but not that of dexamethasone, on inducible nitric oxide synthase gene expression and nitric oxide production in murine macrophages. Int J Immunopharmacol 2000;22:765-774.

32 Salzman AL, Linn SC, Szabo C. Progesterone inhibits inducible nitric oxide synthase mRNA expression in human intestinal epithelial cells. Int J Mol Med 2000; 6:209-216.

33 Dormond O, Lejeune FJ, Ruegg C. Modulation of cdk2, cyclin D1, p16INK4a, p21WAF and p27Kip1 expression in endothelial cells by TNF/IFN gamma. Anticancer Res 2002;22:3159-3163.

34 Haynes MP, Li L, Sinha D, et al. Src kinase mediates phosphatidylinositol 3-kinase/Akt-dependent rapid endothelial nitric-oxide synthase activation by estrogen. J Biol Chem 2003;278:2118-2123.

35 Martin JH, Begum S, Alalami O, et al. Endothelial nitric oxide synthase: correlation with histologic grade, lymph node status and estrogen receptor expression in human breast cancer. Tumour Biol 2000;21:90-97.

36 Zeillinger $\mathrm{R}$, Tantscher E, Schneeberger C, et al. Simultaneous expression of nitric oxide synthase and estrogen receptor in human breast cancer cell lines. Breast Cancer Res Treat 1996;40:205-207.

$37 \mathrm{Ou} \mathrm{J}, \mathrm{Ou} \mathrm{Z}$, Ackerman AW, et al. Inhibition of heat shock protein 90 (hsp90) in proliferating endothelial cells uncouples endothelial nitric oxide synthase activity. Free Radic Biol Med 2003;34:269-276.

38 Tschugguel W, Knogler W, Czerwenka K, et al. Presence of endothelial calcium-dependent nitric oxide synthase in breast apocrine metaplasia. $\mathrm{Br} \mathrm{J}$ Cancer 1996;74:1423-1426.

39 Garban HJ, Bonavida B. Nitric oxide sensitizes ovarian tumor cells to Fas-induced apoptosis. Gynecol Oncol 1999;73:257-264.

40 Horita K, Inase N, Miyake S, et al. Progesterone induces apoptosis in malignant mesothelioma cells. Anticancer Res 2001;21:3871-3874.

41 Gompel A, Somai S, Chaouat M, et al. Hormonal regulation of apoptosis in breast cells and tissues. Steroids 2000;65:593-598.

42 Kloke O, Klaassen U, Oberhoff C, et al. Maintenance treatment with medroxyprogesterone acetate in patients with advanced breast cancer responding to chemotherapy: results of a randomized trial. Essen Breast Cancer Study Group. Breast Cancer Res Treat 1999;55:51-59.

43 Focan C, Beauduin M, Salamon E, et al. Adjuvant high-dose medroxyprogesterone acetate for early breast cancer: 13 years update in a multicentre randomized trial. Br J Cancer 2001;85:1-8.

44 Franke HR, Vermes I. The effect of continuous combined 17beta-oestradiol and dihydrodydrogesterone on apoptotic cell death and proliferation of human breast cancer cells in vitro. Eur J Cancer 2002; 38(Suppl 6):S69-S70. 